\title{
New Concepts Targeting the Biological and Quantum Connections in the Action Mechanism of the Gemmmotherapy
}

\author{
Mihai Surcel $^{1}$, Mioara Butan ${ }^{2}$, Didi Surcel ${ }^{2}$ \\ ${ }^{1}$ Department of Obstetrics and Fetal-Maternal Medicine, Reproductive Biology Unit, Iuliu Hatieganu University of Medicine and Pharmacy, \\ Cluj Napoca, Romania \\ ${ }^{2}$ Department of Immunology, Blue Life Medical Center, Cluj-Napoca, Romania
}

Email address:

mihai_surcel@yahoo.com (M. Surcel)

\section{To cite this article:}

Mihai Surcel, Mioara Butan, Didi Surcel. New Concepts Targeting the Biological and Quantum Connections in the Action Mechanism of the Gemmmotherapy. Biomedical Sciences. Vol. 7, No. 2, 2021, pp. 53-59. doi: 10.11648/j.bs.20210702.13

Received: January 22, 2021; Accepted: February 16, 2021; Published: May 26, 2021

\begin{abstract}
Current frontiers in the biological sciences require an interface between the disciplines of biology and quantum physics to obtain new paradigms in the field of medical practice. Gemmotherapy is a more recent component of phytotherapy, which uses the embryonic tissues of plants, useful for their ability to detoxify cells, drainage of toxic products and for their anti-inflammatory effects. Starting from the fact that gemmotherapy is a type of energy medicine, we focused on some aspects regarding recent acquisitions related to energy and information. The primary element of the universe is energy, the carrier of information, and not material, and space is neither empty nor passive, but is full of virtual energies and information. Traditional medicine and scientific discoveries, from the last 100 years, have demonstrated the existence of a non-material fundamental force that supports all living organisms, namely the biofield. The biofield coordinates all cellular processes / DNA synthesis, RNA, cell division, cell differentiation, morphogenesis, psycho-neuro-endocrine-metabolic-immune regulation, biochemical reactions, the self-regulation / healing / and regeneration processes, which are under the direct command of the energy matrix. Biology is governed by the quantum process, and at its center is DNA. So we can say that man is an energy-informational entity, and the presence of coherent biofields indicates healthy organisms, while biofields affected by chaotic signals indicate diseased organisms. Gemmotherapy, like other types of alternative medicine, is guided by a deep knowledge of natural laws and aims to restore the dynamic energy balance, the basis of healing being provided by the living structures of plants. Having as intervention tool the body's own immune system, therapy with gemmotherapy extracts ensures the improvement of intercellular communication, according to the body's needs, through psycho-neuro-endocrine-immunological mechanisms, correcting the energy behavior of altered tissue, and thus biochemical reactions can be restored normal. Gemmotherapy restores the energy-informational matrix of the body by activating the energy control centers, stimulating the natural formation of stem cells and at the same time by stimulating the recovery processes of damaged and senescent tissues / cells/.
\end{abstract}

Keywords: Gemmotherapy, Quantum Physics, Biofield

\section{Introduction}

Gemmotherapy is a more recent component of phytotherapy, which uses the embryonic tissues of plants, respectively tissues in the cell division phase, known as meristems / gemmoderivates /, very useful for their cellular detoxification capacity, for the drainage of toxic products and not in lastly for their anti-inflammatory, antioxidant, antimicrobial, healing effects, which ensure the restoration of the functionality of the affected tissues [1].

Gemmotherapy is the deep expression of our intrinsic connection with nature, and the major argument in this regard is the healing induced by the meristem, which through the contained elements, similar to those of the human body, brings specific information, energy and bioactive substances to the sick body [1].

The buds of the plants develop in autumn, during the winter they store in them a lot of energy, vital force, vigor, 
organic and inorganic substances, with structures similar to the human body, and during the spring they unfold offering gemmotherapy a raw material of the highest quality [1]. Starting from the fact that Gemmotherapy is a type of energy medicine, we will focus on some aspects regarding recent acquisitions related to energy and information.

It is difficult to fully understand the meaning of a symptom or to understand a disease, when the basis of medical knowledge is not supported by an energetic conception of life [5-7].

From the beginning, man has struggled to find out and understand what life breathes - something that penetrates the physical body from birth to death. Often, questions that concerned him, respectively, how life begins, how we think, why we get sick, what happens after we die, have gone unanswered.

\section{Energy Concept of Life}

Currently, science is undergoing a fundamental paradigm shift. The paradigm that is still dominant today and that describes material objects separated from each other or associated by mechanistic relations of cause and effect, is bankrupt.

The conception of classical science regarding the universe proved to be erroneous. The primary element of the universe is energy, the carrier of information, and not matter, and space is neither empty nor passive, but is full of virtual energies and information [2]. It is becoming increasingly clear that the universe is an integral system of evolution, interconnected and extremely coherent $[6,7]$. All matter has its origin in a FORCE and exists only by virtue of it. We must admit that behind this force there is a Conscious and Intelligent Mind. This Conscious and Intelligent Mind is the Matrix of All Matter," said Max Planck in 1944 [3]. With these words, Max Planck, the father of quantum physics described a universal energy field, which connects everything in Creation. The whole universe is based on a great boiling energy, a vast quantum field, an ocean of microscopic vibrations in the space between things. Living beings, at the deepest level, are packages of quantum energy, which constantly exchange information with this inexhaustible energy of the quantum field $[4,5]$. The subtle entanglement of the 3 worlds - matter, consciousness, energy, welded in one unit, frames man in the universe.

\subsection{The Biofield - The Fundamental Immaterial Force that Controls All Biological Processes of Living Organism}

A substructure of the quantum field, the morphogenetic field is the basis of communication in our universe, stating that it is essentially an environment that records everything, providing a major, fast and efficient means of communication of a being with any other similar being $[6,7]$. The energyinformation fields are our bridges with the Universe [2, 3, 5]. Traditional medicine and the scientific discoveries / from the last 100 years / of some high class physicists, have demonstrated the existence of a fundamental non-material force that supports all living organisms, namely the biofield $[3,7,8]$.

The biofield controls the biochemical reactions, respectively the processes from the material / physical plane, establishes the pattern and governs the processes of selfregulation / healing / and those of regeneration, which are under the direct command of the energy matrix. Biofield realizes all cellular processes / synthesis of DNA, RNA, cell division, cell differentiation, morphogenesis, psycho-neuroendocrine-metabolic-immune regulation. So, biology is governed by the quantum process, and at its center is DNA [7, 9]. It should be noted that only 3\% of DNA human encodes the physical body, the rest, so most DNA is just a receptor of electromagnetic frequencies specific to the morphic field, notes Sheldrake. The morphogenetic field is the general pattern of human finites, but the principle of the morphic field operates identically throughout the Universe.

$\mathrm{He}$ is the one who gives shape to the organs, their qualities and characteristics, the individual characteristics taken from the parents, partially / at least / are taken from the morphic field. So we can say that man is an energy-informational, multi-dimensional entity and that the presence of coherent biofields indicates a healthy organism, while biofields affected by chaotic signals indicate diseased organisms.

In recent years, researchers in multidisciplinary teams have discovered that living cells have membrane organic structures with liquid crystal (CL) properties, as well as semiconductortype molecular systems, which include: water - molecular oxygen - phosphate (PWO), complex, which represents the defining attribute of the life. It should be emphasized that these structures represent the active functional substance for all systems, biological or vegetable, animal or human, allowing communication between them.

These elements underlie bioelectronics, a new science that seeks to explain a particular type of intra-and intercellular electronic communication. It is in our power to know and apply this knowledge, which can be useful in the healing process and in maintaining good health.

Gemmotherapy remedies work in extremely low doses and do not use the same receptor cell activation pathways as allopathic therapy. Gemmotherapy remedies activate cellular energy control systems, which address the whole body, through a resonance mechanism between the field of the remedy and the field of the diseased cell. Following this resonance, the affected cellular energy matrix is restored.

\subsection{Biophotons Emission}

Living things emit weak radiation and this is the most important aspect of biological processes because information about all aspects of life, from cellular communication to the wide range of DNA commands, is based on the exchange of information at the quantum level [8]. A good knowledge of these fundamental energy networks opens a door to unsuspected therapeutic possibilities. Any living organism is an open thermodynamic system, with a rigorous cyber selfcontrol, in which the biofield is the general control system in any phase of the organism's development $[8,9]$. The emission 
of biophotons is specific to all living organisms and each cell is connected by a coherent radiant field to the field of the whole organism, having the possibility to transmit and receive energy through electromagnetic waves [8]. The emission of biophotons is continuous, rhythmic and closely related to all the biorhythms of the body.

Biophotons bring information about the biological state of tissues and ensure a fast and efficient form of intra and intercellular communication [9, 10]. Plants emit about 100 photons / cm2 / sec, with a wavelength of 200-800nm and high frequency, in the visible spectrum, while humans emit only 10 photons / $\mathrm{cm} 2 / \mathrm{sec}$, with the same frequency spectrum. The emission of biophotons is permanent and varies depending on the cell type. Mesenchymal stem cells, fibroblasts and other multipotent cells are characterized by a much faster and more intense emission of biophotons. It should be mentioned that the meristem, due to its richness in embryonic plant stem cells, has a leading place in the release of biophotons. Coherent radiation, generated after the ordering of biophotons in cellular crystal structures, amplifies and becomes information carriers, being transmitted both intracellularly, inter-cellulary and at a distance, cell is coupled by a coherent radiant field to the field of the whole organism, and the emission of biophotons is rhythmic and closely related to all the biorhythms of the organism $[11,14$, $15]$.

\section{Health and Disease of the Body}

Man is a living collection of cosmic information. A collection whose content is dictated by everything that happens in the Universe. In the past, this collection was called Akasha. It was "read" with care and respect by very wise people. In time, the sages disappeared, and the man lost his "sight" and failed to read this book of life $[5,7]$.

But there were oral transmissions or transcripts and extraordinarily useful testimonies that were not obsolete over time. Nowadays, many pages of this collection have begun to be read by modern man with the help of the microscope, magnetic resonance imaging and many other wonderful analysis tools.

But there are so many pages that still can't be read by these methods. The good news is there, and modern science has begun to shake hands with ancient science. Opposite CERN Geneva, the Subatomic Particle Research Institute is a statue of Shiva, the Vedic principle of transformation. Neuroscience quantum physics, epigenetics have gone hand in hand with the meta-sciences and are quite close to reading the universal collection $[6,8,14]$.

To find out, to know, to understand that we are a microcosm integrated in a macrocosm. Universal rhythms are meant to keep our structure in balance and update it. When we disconnect from these rhythms, errors appear in the manifestation code. If we imagine that these letters represent the DNA code, and hormones have a phenomenal intelligence to read and transmit these words and messages to every cell in our body, these will know how to behave.
Hormones do not dictate these messages, they just transmit them. Following these messages, in relation with metabolism, behavior, relationships of all kinds, our way of seeing the world, the response to life we live is overwhelmingly influenced. The boundary between an organic and a functional disease is becoming increasingly difficult and blurred in the context of moving away from the old knowledge of the "global man", and moving towards its partial appearance is now a process of obsessive search, highly specialized and modernly instrumented of highperformance equipment, through a sustained intellectual effort, focused on organic and cellular pathology.

Our ancestors perceived the world as an interdependent whole. Today, ancient healing techniques are beginning to gain ground, as they continue the holistic tradition, in which the disease is considered a blockage or imbalance in the energy flow of a vital force.

Being healthy does not only mean the absence of any symptoms or disease, but also the balance of all functions of the physical body, namely the harmonious state of vital energies. Whether or not diseases manifest in the physical body, they always have a previous cause, of an energetic nature, which is usually unnoticeable through our senses, and is most often preceded by a mental or emotional cause.

Knowing and removing in time the true causes of diseases, which are energetic in nature, will regain immunity to external aggressive factors and avoid the formation of pathogenic energy abnormalities and their subsequent manifestations, which would take the form of various diseases of the physical body. The quality and duration of our health are the direct result of how well our biofields are tuned with the universal rhythms and how efficiently we have taken the following steps:

1. Let's get rid of stress;

2. To amplify or create feelings of peace and deep inner peace;

3. To clean all the cellular structures of "rust", like pollutants at the level of resp tract/ smoking, respiratory toxins, and at the level of digestive tub (alcohol, unhealthy foods), drugs

4. To get rid of negative emotional baggage.

If these ways of maintaining health are exhausted, then that energy imbalance is installed and the first to respond to the impasse reached is the immune system, which is involved in restoring health, with a whole army of biologically active cells and, specific and non-specific molecules [1, 12]. Deep, energetic / molecular and functional healing is based on protein metabolism, which is the common denominator of all reactions induced for healing. Integrity of communication elements of this triad - immune system, vascular system, mesenchymal system means health, adaptation to everything around us from the internal and external environment, and people who enjoy the proper functioning of this network are considered people with good immunity. 


\section{Characteristics of Gemmotherapy Remedies}

Gemmotherapy is a complex biological system, a system of dynamic natural elements, which behaves like a living, undefined, infinite and ubiquitous organism, composed of thousands of active molecules, which ensures its ability to perform a multitude of functions through cooperation. synergistic and in perfect harmony of the elements contained (Fernando Pitera). This entity, unitary and multiple at the same time, has as a major feature the presence of plant stem cells with high capacity for self-regeneration and differentiation, which is never complete, so part of the meristem remains in continuous regeneration, and also has a high capacity to emit quickly and intensely an important level of biophotons, suggesting an important energyinformational availability in the process of adaptation, evolution and recreation of the whole plant. It must be emphasized that by intervening to separate these extremely dynamic elements, the gemoderivate loses its essential effect, which gives it a very special feature, characterized by the notions of infinity, indefinite and undifferentiated, and thus its magical power would disappear. The major feature of the gemmotherapy is that at this level, the boundaries of the biological sciences impose an interface between the disciplines of biology, chemistry, physics, biophotonics and immunology to obtain new paradigms in the medical fields. In Vibrational Medicine, information is represented by the symptom, which is not only the "sign" of an organic or functional alteration of a tissue or organ, but also brings information on the particular way of expressing energy throughout the body. In other words, the symptom, the syndrome and the disease represent the functional and structural expression of the centers of self-regulation and self-organization of vital energy, which tries to overcome a state of suffering of a system, and through this attempt to heal tries to increase the state of "order" that goes beyond the state of "chaos", through which it passes at that moment. When chaos (biochemical, cellular, etc.) is reconverted in order, healing occurs, and when this does not happen. the system loses energy and the disorganization extends to the subsystems with the appearance of chronic pathology. When the system no longer responds to the initial therapeutic regulation of symptoms, the disorder goes deep, and the late effects are difficult to control. So, from an energetic point of view, the disease is an attempt towards a new order, approached to avoid the destruction of the living system. The disease has a causal coherence, in which the symptom is the expression of adaptation to a new order, which is to be established. Whatever the therapeutic attempt to block the symptoms, without interpreting the signal from the balance of the functional order, only produces subsequent phenomena of entropic self-regulation of energy, each time deeper, more serious, with variable latency and not it is also related to the initial suppressive therapeutic act. In other words, the suppression of signals aggravates the pathological process; from functional it passes to a deeper lesional level, and the disorder initially includes the cytoplasm, so that later it also reaches the cell nucleus. Only the understanding of the phenomenon through the energetic interpretation and by the administration of another analogical signal, able to modify the functional order of the system, can achieve the restoration of the balance and of the health $[1,4,7]$.

\subsection{The Mechanism of Action of Gemmotherapy}

Gemmotherapy, like other types of alternative medicine, is guided by a deep knowledge of natural laws and has the major goal of restoring the dynamic energy balance, the basis of healing being provided by the living structures of plants. Gemmotherapy works in homeopathic doses and does not use the same receptor cell activation pathways as allopathic medication. The essence of gemmotherapy, respectively of the meristem therapy, means a profound interference of aberrant metabolic circuits, which no longer meet the need to adapt the body to the internal and external environment.

Gemmotherapy remedies activate cellular energy control systems, which address the whole body, through a resonance mechanism between the field of the gemmotherapy remedy and the field of the diseased cell. Following this resonance, the cellular energy matrix of the diseased cell is restored through a constructive resonance mechanism.

Considering the disease state a deviation from the universal standard of vibration, characteristic for healthy cells, the gemmotherapy resonates with the cellular energy fields and brings them back within the limits of the vibrational matrix, characteristic of the state of health. Having as intervention tool the body's own immune system, therapy with gemmotherapy extracts ensures the improvement of intercellular communication, according to the body's needs, through psycho-neuro-metabolicendocrine-immunological mechanisms, correcting the energetic behavior of altered tissue, and thus biochemical reactions can be restored at the normal level [13].

Gemotherapy promotes the regulation of the metabolic balance (microelements, hormones, vitamins) and finally ensures the repair, regeneration, restructuring and functioning of the damaged tissue. Gemotherapy intervenes in maintaining or regaining health or in maintaining it by intervening in initiating defense reactions of the body at the cellular level, rearrangement of intercellular communication, which is also ensured by Toll-like $\mathrm{R}$ receptors, present in both plants and humans with similar structures. elimination of harmful foreign substances, toxic, cellular detritus by ensuring efficient drainage. Improving cellular $\mathrm{pH}$ by suppressing acidosis induced in the inflammatory process, more obviously in the chronic one. Gemmotherapy remedies thus become field re-ordinators, which transfer their vibrational energy to the energy field of the diseased cell.

The action of gemmotherapy profound, and the antioxidant, immunomodulatory, anti-inflammatory, anti-infective effect, supported by the promotion of effective drainage extends in the case of meristems to antitumor effects (Buxus, Vasc, Dandelion, Vine).

The improvement of the investigated biochemical, 
immunological and histological parameters are evidence for the return of the functions of the affected tissues, which were no longer able to cope with the activities for which they were programmed.

\subsection{Beneficial Effects of Gemootherapy}

The human body is a complex biological structure, which we can appreciate as perfect, given its ability to adapt and survive in unfavorable external and internal conditions, due to its endowment with self-regulation and self-cleaning systems, systems necessary for detoxification, for maintaining homeostasis, for recovery, and regeneration.

Until recent years, allopathic and even natural treatments considered that there were deficiencies in some elements in the body, such as calcium, magnesium, iron, and that the suffering given by their lack could be corrected by dietary supplements.

Currently it is a paradigm shift, which starts from the premise that in the human body there is too much waste, residues, substances that must be discharged to allow the body to fulfill its self-regulatory functions.

The presence of these own wastes of the body are the real causes, which trigger the lack of minerals such as calcium, magnesium, etc., given their use by the body loaded with waste, to neutralize acid residues. So the new paradigm proposes the transition from focusing on "deficiencies" to "excesses", too many toxins brought and remaining in the body through the digestive tract, through diet, respiratory and skin and not to omit too many toxins. from excessive activity and the exaggerated and prolonged response of the body exposed to stress. Pol Henry, pioneer in GEMMOTHERAPY, demonstrated 50 years ago, the efficiency of therapy with extracts from young plant tissues, which bring with them the dynamics and vitality of the plant kingdom, intricate with an extraordinary force of expansion and multiplication of stem cells. plant, which through the unique capacity of incomplete differentiation, characteristic of all-powerful cells, ensures a continuous self-regeneration.

In the context of this excess waste in the body, gemmotherapy treatment is welcomed precisely by its ability to remove these toxic residues, both directly and indirectly by helping the main organs whose role is to eliminate residues from the body.

\subsection{Detoxification Is a New Treatment Paradigm in Recent Natural Medicine and Occupies a Special Place in Gemotherapy}

It starts from the idea of the existence of blockages in the way of elimination through the body's own elimination organs, both its own waste and those that entered the body accidentally. It should be mentioned that lymph is the fluid, which cleans the residues and wastes from the cells and eliminates them outside the body through the kidneys and intestines. In the new paradigm of detoxification, the lymphatic system has an essential place in disease states, because it presents itself as a system of "sewerage" clogged with acid residues, and they flow into tissues, irritate them and induce an inflammatory response in the affected area. It should be noted that the main "gates" for discharging waste from the lymphatic system are the digestive tract, kidneys and skin. The detoxification strategy consists in the use of gemmotherapy extracts, which aim at improving the function of these organs directly involved in the cleaning process. Periodic detoxification to avoid systemic acidosis is achieved through "preventive" procedures during the changing seasons, spring detoxification being the most important. In chronic diseases, the real detoxification, sustained with gemmoderivates, which aims both at the elimination of toxic residues and at supporting the drainage function of the lymphatic system, is of real use. And in this case, gemmotherapy have an important role through their ability to act antioxidant, antibacterial, antifungal, antiviral, antiinflammatory, antispasmodic, antiallergic, decongestant, healing, anti sclerosis, etc., with deep involvement in the response of aggressive tissue. These possibilities of intervention of gemmoderivato explain their possible beneficial intervention in all stages of the evolution of the acute, subacute, chronic inflammatory process and even in organized inflammatory syndromes. A classification of antiinflammatory gemmotherapy can be noted according to the type of inflammatory process.

In Acute Inflammations the following hemoderivatives are useful: Black alder, White alder, Blackcurrant, Fluffy birch, Rosehip, Poplar, Ash, Blood, Elm.

The following gemmoderivates are useful in Subacute Inflammations: Silver birch, Walnut, Beech. In Chronic Inflammations the following hemoderivatives are useful: Blackberry, Vine, Sequoia, Cranberry, White Birch, Thuja; Vasc, Beech, Taraxacum. The following gemmoderivates are useful in organized inflammatory syndromes and tumor conditions: Boxwood, Dandelion, Mistletoe, Black Grass, Vine, White Alder. Depending on the diagnosis, the age of the disease, the associated comorbidities, the result of laboratory and paraclinical investigations, the doctor has the task of establishing a personalized therapeutic scheme, with gemmotherapy derivatives, the most appropriate for the case. An inadequate Excessive inflammatory response, when external aggressors are no longer present, leads to Autoimmunity and the development of a chronic inflammatory process, respectively $[6,12]$.

In these situations, too, a careful, but easy, longer-lasting immunological regulation is required, without causing an alert rhythm of intervention, avoiding excessive tissue destruction and returning to tissue normality, and therapeutic strategies with meristematic preparations place gemmotherapy in the leading place.

Adaptation is also present in pathological conditions. in a broad sense, adaptation is seen as the adjustment of the organism to all the conditions that ensure the survival of that organism.

The doctor often faces the situation when the patient is adapted, but is still ill (eg condition after nephrectomy, aortic stenosis). 
These situations in which there is still adaptation to the environment, is another type of adaptation, respectively compensatory adaptation, process of counterbalancing a deficiency or maintaining vital functions. In case of injuries, or serious functional disorders of some organs or systems, a survival framework is created as in any kind of adaptation, but with a more important energetic effort. Depending on the diagnosis, the type and age of the condition, the results of laboratory and paraclinical investigations and other associated comorbidities, the doctor will determine the most appropriate combination of gemmotherapy derivatives. Gemmotherapy, like other types of alternative medicine, is guided by a deep knowledge of natural laws and has as its major goal the restoration of the dynamic balance existing at the base of the living structures of plants. The essence of gemmotherapy, meristem therapy, is a profound interference of aberrant metabolic circuits, which no longer respond to the need to adapt the body to the internal and external environment.

\section{Basic Biological Regulation System BBRS}

The Basal System of Biological Regulation, respectively The Extracellular Matrix (ECM) penetrates the entire body and contacts the organs in the body more than any other tissue. Its function, which consists of transmitting nerve, immunological, hormonal and nutritional impulses to the body's tissues, has been overlooked in conventional medicine [15].

Currently, the role of the extracellular matrix in regulating physiological functions, dysfunction problems induced by the deposition of toxins and other forms of tissue injury is emphasized, and last but not least, the involvement of possible mechanisms induced by associated alternative therapies. The connective tissue is the true "basal regulation system", which ensures the basic physiological regulation of the body [15].

All changes in the body induced by the influences of the external and internal environment are achieved at this level by the participation of numerous biologically active cells and molecules.

The central position in the extracellular matrix is held by the components of the immune system and among the basic elements we note membrane receptors, capillaries, neurovegetative terminations, endocrine hormones, cytokines, chemokines, growth and signaling factors, and by cellular mechanisms that are induced communication between cells and the external environment.

Communication between cells and the external environment is performed by extracellular vesicles (ECV) and endosomes and is performed at the level of the extracellular matrix (ECM) [13].

A large amount of information can be stored in the extracellular matrix and transmitted to cells in the form of instructions for physiological activity / regulation of cells [7].

Psycho-neuro-endocrine-immunological information is also transmitted at this level; they coordinate and control the activity of cells through messenger molecules equipped with cellular receptors.

The accumulation of stress factors (physical or biochemical) in this place represents the triggering potential for the onset and progression of pathological processes.

BBRS is inseparably linked to therapeutic regulatory medicine. Even modern medical perspectives in regulatory medicine can only be understood if we become aware of this major body system, the BBRS $[14,15]$. Regulation is the key word in the frontiers of medicine today. Gemmotherapy together with all forms of alternative medicine are based on this biological regulatory mechanism.

Today, we know that the constituents generated in the process of disease development and healing are transferred through the nerves of the autonomic nervous system, through the hormonal and immune system. The body's organs and cells are not directly related to the blood, not even the smallest blood vessels or nerves that will end up in the transit mesenchyme or extracellular matrix [14].

These exchanges take place, according to Pisschinger, through the so-called "fundamental system" of soft tissues and extracellular fluid. BBRS regulates the main basic processes of life, such as metabolism, blood flow, temperature, respiration, energy metabolism and acid-base balance [14].

The connective tissue of the extracellular matrix is the most forgotten organ, although it holds $60 \%$ of body weight. It is unanimously accepted that "LIFE" is Organized and Self-Regulated Energy, but self-regulation and organization will be possible only through the exchange of information, and this exchange of information is exactly the mechanism of action of the BBRS [15]. So for life "it takes matter, energy and more especially the exchange of information.

The causes that direct the doctor to a natural therapy can be summarized as follows:

The boundary between an organic and a functional disease becomes more and more difficult and blurred in the context of moving away from the old knowledge of the "global man" and moving towards its partial aspect, achieved by obsessive, highly specialized search, modernly instrumented by highperformance equipment. the intellectual effort focused on the organic and cellular pathology of the last century.

Globality has been replaced by detail, the particular, the syndrome. Therapy according to this concept has become increasingly anti symptomatic and dangerous, and the therapeutic effect and side effects increasingly subtle. The therapeutic effect is always based on symptomatic suppression, achieved through pre-established therapeutic schemes and protocols. Individual reactivity is not even taken into account; the suppression of the body's defensive response is completely ignored. Whenever the therapeutic action detached from this conception inevitably becomes harmful, and in time, palliative or suppressive.

\section{Conclusions}

Gemmotherapy falls into the category of future highperformance therapies, given the ability of meristems to 
intervene regulator, through energy mechanism, in the complex network of cells and biologically active molecules, derived from the 4 major mesenchymal systems (mesenchymal stem cell system, immune system, the vascular system and the nervous system), thus ensuring the energetic harmony between the compartments of the systems involved with the restoration of cellular communication, the energetic, functional and morphological recovery of the affected tissue and finally with the restoration of the organism in harmony with the environment. The unique properties of meristems are those that give gemmotherapy systemic actions, necessary to rebalance the vast network of interconnections, which is imposed as a basic element in the functioning mechanism of the complex psycho-neuroendocrine-immune-metabolic system (PNEIM). Given that the cells in the body are subjected to the action of interrupting agents of intercellular informational messages, the use of gemmotherapy products demonstrates that the remedy of altered intercellular communication ensures the maintenance / restoration of internal homeostasis. The meristem, together with the body structures majorly involved in remedying the impact, respectively, the mesenchymal system (MSC), the immune system (HSC), the reticuloendothelial system (SRE) and other complementary compartments, represent the direct effector elements, continuously adapting to environmental conditions., who join their forces in order to restore the functional and structural harmony of the affected organ, ensuring the deep, energetic / molecular and functional healing of the injured territory.

Finally, we can emphasize the preventive role of gemmotherapy extracts, precisely through this subtle mechanism of remediation of energy disorders, which, when neglected, can generate, after prolonged exposure, severe morphological damage.

Gemmotherapy, through its meristematic remedies ensures a gentle, logical, effective therapeutic act, which does not oppose the natural, biological mechanisms of healing and at the same time is free of unwanted side effects.

\section{References}

[1] Marcello Nicoletti e Fernando Piterà di Clima Gemmoterapia Fondamenti Scientifici della Moderna meristemoterapia (Italiano) 2018.
[2] Lipton B. H. - Quantum Physics and Creation, Ed. For You, Bucureşti., 2008.

[3] Lynne McTaggart - The field. The search for the secret force of the Universe Adevarul Divin, 2009.

[4] Greg Braden The Spontaneous Healing of Belief: Shattering the Paradigm of False Limits Apr 1, 2009.

[5] Ervin Laszlo - Science and the Akashic field https://www.jovis.ro/carti-fizica-cuantica/stiinta-campulakashic-4390.htm.

[6] Traian Trandafir, Mihaela Gheorghiu- Introduction to Vibrational Medicine, 2009.

[7] Dumitru Constantin Dulcan - In Search of the Lost Meaning2008.

[8] Fritz - Albert Popp - Biophotonen - Neue Horizonte in der Medizin, 1, 12. 2006.

[9] Roeland Van Wijk, Eduard P. A. Van Wijk - BBRS Biophotons in Diagnostics Progress and Expectations Amersfoort, The Netherlands. The following... The concept of BBRS is rooted in fruithttps://pdfs.semanticscholar.org/.../1c50c8a67c2321a64fa1c.

[10] TM Srinivasan -Biophotons as Subtle Energy Carriers, Int J

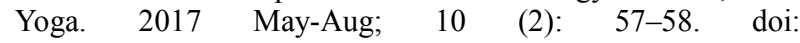
10.4103/ijoy.IJOY_18_17, PMCID: PMC5433113 PMID: 28546674.

[11] Beverly Rubik, Harry Jabs- Effects of intention, energy healing, and mind-body states on biophoton emission- Cosmos and History: The Journal of Natural and Social Philosophy, vol. 13, no. 2, 2017, www.cosmosandhistory.org 227.

[12] Nauta A. J, Fibbe W. E - Immunomodulatory Properties of MSC, Blood. 2007; 110 (10): 3499-506, MSC, Blood. 2007; 110 (10): 3499-506.

[13] Jong-Kuen Lee, Ji-Young Jang, Yoon-Kyung Jeon ${ }_{2}$ Chul-Woo Kim_Extracellular vesicles as an emerging paradigm of cell-tocell communication in stem cell biology, 2014.

[14] HALLMANN R, ZHANG X, DI RUSSO J, LI L, SONG J, HANNOCKS MJ, et all.-The regulation of immune cell trafficking by the extracellular matrix.- ACS Biomater. Sci. Eng., 2018, 4 (4), pp 1208-1222 DOI: $0.1021 /$ acsbiomaterials. $7 \mathrm{~b} 00083$.

[15] RON ZOET, Bert Pijnenburg and Hugo Nielsen - BBRS Science and Medicine Biocommunication on and the Basic Biological Regulatory System the scientific basis, 2010. 